\title{
Dual Characterizations of Set Containments with Strict Convex Inequalities
}

\author{
M.A. Goberna*, V. Jeyakumar ${ }^{\dagger}$ and N. Dinh \\ Revised Version:October 14, 2008
}

\begin{abstract}
Characterizations of the containment of a convex set either in an arbitrary convex set or in the complement of a finite union of convex sets (i.e. the set, described by reverse-convex inequalities) are given. These characterizations provide ways of verifying the containments either by comparing their corresponding dual cones or by checking the consistency of suitable associated systems. The convex sets considered in this paper are the solution sets of an arbitrary number of convex inequalities, which can be either weak or strict inequalities. Particular cases of dual characterizations of set containments have played key roles in solving large scale knowledge-based data classification problems where they are used to describe the containments as inequality constraints in optimization problems. The idea of evenly convex set (intersection of open half spaces), which was introduced by W. Fenchel in 1952, is used to derive the dual conditions, characterizing the set containments.

Key words: Set containment, convex functions, semi-infinite systems, existence theorems, dual cones, conjugacy.
\end{abstract}

\section{Introduction}

Consider the sets

$$
F:=\left\{x \in \mathbb{R}^{n} \mid f_{t}(x)<0, \forall t \in S ; f_{t}(x) \leq 0, \forall t \in W\right\}
$$

*Department of Statistics and Operations Research, University of Alicante, 03080 Alicante, Spain. E-mail: mgoberna@ua.es. Research of this author was supported by MCYT of Spain and FEDER of UE, Grant BMF2002-04114-CO201

$\dagger$ Department of Applied Mathematics, University of New South Wales, Sydney, Australia. Email: jeya@maths.unsw.edu.au

$\ddagger$ Department of Mathematics-Informatics, Ho Chi Minh City University of Pedagogy, 280 An Duong Vuong Street, Distr. 5, HCM city, Vietman. E-mail: ndinh@hcmup.edu.vn. Work of this author was carried out while he was at The University of New South Wales. 
and

$$
G=\left\{x \in \mathbb{R}^{n} \mid g_{i}(x) \leq 0, \forall i \in I ; h_{j}(x) \geq 0, \forall j \in J\right\},
$$

where $S \cap W=\varnothing, S \cup W \neq \varnothing, I \cap J=\varnothing, I \cup J \neq \varnothing$, and all the functions, $\left\{f_{t}, t \in S \cup W\right\},\left\{g_{i}, i \in I\right\}$, and $\left\{h_{j}, j \in J\right\}$, are convex functions from $\mathbb{R}^{n}$ to $\mathbb{R}$. The set containment problem that is studied in this paper, consists of deciding whether $F \subset G$ or not. Dual characterizations of such set containments have played a key role in solving large scale knowledge-based data classification problems where they are used to describe the containments as inequality constraints in optimization problems (see e.g., [2, 10, 11] and [8]). For instance, the incorporation of prior knowledge in the form of a polyhedral knowledge set in the construction of a linear classifier is modelled as the set containment $F \subset G$ [2], where $F$ is a given polyhedral convex set and $G$ is a given closed halfspace. The dual characterizations of the set containment were obtained using the classical nonhomogeneous Farkas Lemma [9].

More recently, various extensions of the containment problem to more general situations have been obtained in [8] and [11], by means of mathematical programming theory and conjugacy theory, respectively, where $S=\varnothing$ (i.e. without strict inequalities). In this paper we establish dual characterizations by allowing the systems defining $F$ and $G$ to contain strict inequalities, as depicted in Figure 1 below. Such kind of systems also arise naturally in the characterization of the stable containment; i.e., establishing conditions which guarantee that the inclusion is preserved under sufficiently small perturbations of the systems representing $F$ and $G$.

$$
\begin{gathered}
(0,0)(8,7.5)[\text { linestyle=dashed }](0,5.5)(5.2,6.9)(-.5,1.9)(1.9,6.8) \\
(8.2,1.9)(3,7.5)(3,1.5) 345130[\text { linestyle=dashed] }(4.2,2.8) 3130220 \\
{[\text { linestyle=dashed }](2,2.8) 332060(3.8,3.4) 3200250(3,4.1) \mathrm{F}(6.5,2) \mathrm{G}} \\
(5.7,6) u_{i}(x)=\alpha_{i}(3.1,3.1) f_{t}(x)<0, t \in S(3.1,2.2) f_{t}(x) \leq 0, t \in W
\end{gathered}
$$

Figure 1: Containment of the evenly convex set $F=\left\{x \mid f_{t}(x)<0, \forall t \in\right.$ $\left.S ; f_{t}(x) \leq 0, \forall t \in W\right\}$ in the polyhedral set $G=\left\{x \mid u_{i}(x) \leq \alpha_{i}, \forall i \in\right.$ $\left.I, u_{i}(x)<\alpha_{i}, \forall i \in J\right\}$, where $f_{t}: \mathbb{R}^{n} \rightarrow \mathbb{R}$ is a convex function and $u_{i} \in \mathbb{R}^{n}$ and $\alpha_{i} \in \mathbb{R}$.

The main basic tool in our approach in deriving the dual characterizations is the association of two dual cones in $\mathbb{R}^{n+1}$, say $K$ and $M$, such that $F \subset G$ if and only if $M \subset K$. Since $M \subset K$ can be interpreted as a dual condition, the verification of the containment reduces to the effective calculus of the corresponding dual cones. In the case where $F$ is the intersection of a family of open convex sets, $\left\{x \in \mathbb{R}^{n} \mid\right.$ $\left.f_{t}(x)<0\right\}, t \in S$, with a family of closed convex sets, $\left\{x \in \mathbb{R}^{n} \mid f_{t}(x) \leq 0\right\}, t \in W$, $F$ turns out to be an evenly convex set (i.e., the intersection of open halfspaces, see [1]), represented by means of a convex inequality system. The dual cones of closed convex sets were introduced in [3] in order to characterize large classes of closed 
convex sets from a geometric point of view. The dual cones of evenly convex sets that are introduced for the first time in the present paper, play a central role in describing the dual conditions.

The paper is organized as follows. Section 2 contains the necessary notation and some basic results on convex as well as evenly convex sets to be used later. Section 3 develops calculus rules for the dual cone of a closed convex set. Section 4 considers stable containment of closed convex sets. Section 5 defines dual cones for evenly convex sets and develops calculus rules which are similar to those obtained in Section 3. These cones provide dual characterizations of containment for convex sets which are represented by means of strict constraints. Finally, Section 6 presents general existence theorems for several classes of convex systems which contain strict inequalities.

\section{Preliminaries: Evenly Convex Sets}

All the vectors in $\mathbb{R}^{n}$ will be interpreted as column vectors. The inner product of two vectors $u$ and $x$ will be denoted by either $u^{\prime} x$ or $u(x)$, and the Euclidean distance between $u$ and $x$ will be denoted by $d(u, x)=\|u-x\|$. Given a set $X \subseteq \mathbb{R}^{n}$, we shall denote by int $X, \operatorname{bd} X, \operatorname{cl} X, \operatorname{co} X$, and coneco $X$ the interior, the boundary, the closure, the convex hull and the convex cone generated by $X$ respectively. By $\mathbb{R}_{+}$ and $\mathbb{R}_{++}$we denote the sets of nonnegative and positive real numbers, respectively, so that $\mathbb{R}_{+} X:=\{\lambda x \mid \lambda \geq 0, x \in X\}$ and $\mathbb{R}_{++} X:=\{\lambda x \mid \lambda>0, x \in X\}$ are cones in $\mathbb{R}^{n}$, with the null vector $0_{n} \in \mathbb{R}_{+} X$. The smallest convex cone containing $X \cup\left\{0_{n}\right\}$ is coneco $X=\mathbb{R}_{+} \operatorname{co} X$.

Fenchel [1] defined the class of evenly convex sets as the intersections of open halfspaces. The set $C$ is evenly convex if and only if for all $x \notin C$ there exists a hyperplane $H$ such that $x \in H$ and $H \cap C=\varnothing$. The evenly convex hull of $X[1]$, denoted by eco $X$, is the smallest evenly convex set which contains $X$ (i.e., it is the intersection of all the open halfspaces which contain $X$ ). It is known that eco $X$ is obtained by eliminating from $\operatorname{clco} X$ those exposed faces which do not contain points of $X$ (Proposition 2.1 in [4]). From the definition, given $\bar{x} \in \mathbb{R}^{n}, \bar{x} \notin$ eco $X$ if and only if there exists $z \in \mathbb{R}^{n}$ such that $z^{\prime}(x-\bar{x})<0$ for all $x \in X$.

The following existence theorem for linear inequality systems containing strict inequalities will be used later.

Proposition 2.1 (Theorem 3.1 [4]) Let $S$ be non-empty. The system $\left\{a_{t}^{\prime} x<b_{t}, t \in\right.$ $\left.S ; a_{t}^{\prime} x \leq b_{t}, t \in W\right\}$ is consistent if and only if

$$
0_{n+1} \notin \text { eco }\left[\left\{\left(\begin{array}{l}
a_{t} \\
b_{t}
\end{array}\right), t \in S\right\}+\mathbb{R}_{+}\left\{\left(\begin{array}{l}
a_{t} \\
b_{t}
\end{array}\right), t \in W\right\} ;\left(\begin{array}{c}
0_{n} \\
1
\end{array}\right)\right] .
$$

The support function of $X$ is $\sigma_{X}(u)=\sup _{x \in X} u(x)$ and the indicator function 
of $X$ is defined by

$$
\delta_{X}(x)= \begin{cases}0, & x \in X \\ +\infty, & x \notin X .\end{cases}
$$

Given a proper convex function $f: \mathbb{R}^{n} \rightarrow \mathbb{R} \cup\{+\infty\}$, the conjugate function of $f$ is $f^{*}: \mathbb{R}^{n} \rightarrow \mathbb{R} \cup\{+\infty\}$ defined by

$$
f^{*}(u)=\sup _{x \in \operatorname{dom} f}\{u(x)-f(x)\}
$$

where $\operatorname{dom} f:=\left\{x \in \mathbb{R}^{n} \mid f(x)<+\infty\right\}$ is the domain of $f$. The epigraph of $f$ is defined by

$$
\text { epi } f=\left\{\left(\begin{array}{l}
x \\
\gamma
\end{array}\right) \in \mathbb{R}^{n+1} \mid f(x) \leq \gamma, x \in \operatorname{dom} f\right\} \text {. }
$$

Many dual conditions are formulated in terms of epi $f_{t}^{*}$, where $f_{t}$ defines a constraint. So, it is important to note that epi $f_{t}^{*}$ can be expressed in terms of $f_{t}$ by exploiting the information at an arbitrary point $\bar{x} \in \operatorname{dom} f_{t}$. In fact, according to Proposition 2.1 in $[8]$,

$$
\text { epi } f_{t}^{*}=\bigcup_{\varepsilon \in \mathbb{R}_{+}}\left\{\left(\begin{array}{c}
v \\
\varepsilon+v^{\prime} \bar{x}-f_{t}(\bar{x})
\end{array}\right) \mid v \in \partial_{\varepsilon} f_{t}(\bar{x})\right\},
$$

where $\partial_{\varepsilon} f_{t}(\bar{x})$ is the $\varepsilon$-subdifferential of $f_{t}$ at $\bar{x}$, i.e.,

$$
\partial_{\varepsilon} f_{t}(\bar{x})=\left\{v \in \mathbb{R}^{n} \mid f_{t}(x) \geq f_{t}(\bar{x})+v^{\prime}(x-\bar{x})-\varepsilon, \forall x \in \operatorname{dom} f_{t}\right\} .
$$

Recall that the subdifferential of $f_{t}$ at $\bar{x}$ is $\partial f_{t}(\bar{x})=\bigcap_{\varepsilon \geq 0} \partial_{\varepsilon} f_{t}(\bar{x})$.

The following result is fundamental for the characterization of containments of closed convex sets.

Proposition 2.2 (Lemma 3.1 [8]) Let $f: \mathbb{R}^{n} \rightarrow \mathbb{R} \cup\{+\infty\}$ be a proper convex lower semicontinuous (lsc) function, and let $F=\left\{x \in \mathbb{R}^{n} \mid f(x) \leq 0\right\}$. Then the following statements hold:

(i) $F \neq \varnothing$ if and only if $\left(\begin{array}{c}0_{n} \\ -1\end{array}\right) \notin \mathrm{cl}\left(\mathbb{R}_{+}\right.$epi $\left.f^{*}\right)$.

(ii) If $F \neq \varnothing$, then epi $\sigma_{F}=\operatorname{cl}\left(\mathbb{R}_{+}\right.$epi $\left.f^{*}\right)$.

\section{Containments of Closed Convex Sets}

Consider the sets

$$
F=\left\{x \in \mathbb{R}^{n} \mid f_{t}(x) \leq 0, \forall t \in W\right\}
$$

and

$$
G=\left\{x \in \mathbb{R}^{n} \mid g_{i}(x) \leq 0, \forall i \in I ; h_{j}(x) \geq 0, \forall j \in J\right\},
$$


where $W \neq \varnothing, I \cap J=\varnothing, I \cup J \neq \varnothing$, and all the functions, $\left\{f_{t}, t \in W\right\},\left\{g_{i}, i \in I\right\}$, and $\left\{h_{j}, j \in J\right\}$, are convex functions from $\mathbb{R}^{n}$ to $\mathbb{R}$.

Mangasarian [11] presented dual characterizations of the set containment $F \subset G$ in the following cases:

Case 1: $|W|<\infty,|I|<\infty, J=\varnothing$, and all the involved functions are affine (i.e., $F$ and $G$ are given polyhedral convex sets).

Case 2: $|W|<\infty, I=\varnothing,|J|<\infty,\left\{f_{t}, t \in W\right\}$ are affine functions and $\left\{h_{j}, j \in J\right\}$ are quadratic convex functions (i.e., $F$ is a polyhedral convex set and $G$ is a reverseconvex quadratic set).

Case 3: $|W|<\infty, I=\varnothing,|J|<\infty$, and $\left\{f_{t}, t \in W\right\}$ and $\left\{h_{j}, j \in J\right\}$ are differentiable convex functions (so that $F$ is a closed convex set and $G$ is a closed reverse-convex set, both sets defined by means of ordinary systems).

The recent paper [8] established dual characterizations of the containment problem in the following cases:

Case 4: $W$ is arbitrary, $|I|<\infty, J=\varnothing,\left\{f_{t}, t \in W\right\}$ are convex (affine) functions, and $\left\{g_{i}, i \in I\right\}$ are affine functions (i.e., $F$ is the solution set of a convex (linear) semi-infinite system and $G$ is a polyhedral convex set).

Case 5: $W$ is arbitrary, $I=\varnothing,|J|<\infty$, and $\left\{f_{t}, t \in W\right\}$ and $\left\{h_{j}, j \in J\right\}$ are convex functions (i.e., $F$ is as in Case 4 and $G$ is a reverse-convex set described by means of reverse convex inequalities).

We assume that $G$ is represented in a similar way when $J=\varnothing$. In relation to the reverse-convex set $G$ in the Case 5 , let us observe that we can express

$$
G=\left\{x \in \mathbb{R}^{n} \mid h_{j}(x) \geq 0, \forall j \in J\right\}=\mathbb{R}^{n} \backslash \bigcup_{j \in J} G_{j}
$$

where $G_{j}:=\left\{x \in \mathbb{R}^{n} \mid h_{j}(x)<0\right\}$ for all $j \in J$. Obviously, $F \subset G$ if and only if $F \cap G_{j}=\varnothing$ for all $j \in J$, so that the basic problem is to determine the existence of solution of a system similar to (1.1):

$$
\left\{f_{t}(x) \leq 0, t \in W ; f_{t}(x)<0, t \in S ; h_{j}(x)<0\right\} .
$$

Consequently, existence theorems for convex systems possibly containing strict inequalities play a double role in our approach. In fact, they provide tests for (1.1) to be consistent (otherwise the containment problem is trivial) and they provide dual characterizations of $F \subset G$ when $G$ is a reverse- convex set.

We begin by developing calculus rules for the dual cone of a closed convex set, revisiting Case 4 as an immediate application. We define the weak dual cone of the nonempty closed convex set $F \subset \mathbb{R}^{n}$ as

$$
K^{\leq}:=\left\{\left(\begin{array}{l}
a \\
b
\end{array}\right) \in \mathbb{R}^{n+1} \mid a^{\prime} x \leq b, \forall x \in F\right\}=\operatorname{epi} \sigma_{F}
$$


Obviously, coneco $\left\{\left(\begin{array}{c}0_{n} \\ 1\end{array}\right)\right\} \subset K \leq$ and the equality holds if and only if $F=\mathbb{R}^{n}$. It is known that $F$ is bounded if and only if $\left(\begin{array}{c}0_{n} \\ 1\end{array}\right) \in \operatorname{int} K \leq$ (see, e.g. Theorem 9.3 in [5]). The standard hyperplane separation arguments yield

$$
F=\left\{x \in \mathbb{R}^{n} \mid a^{\prime} x \leq b, \forall\left(\begin{array}{l}
a \\
b
\end{array}\right) \in K^{\leq}\right\} .
$$

Observe the symmetry of (3.2) and (3.3): the index set of the linear system in one of the formulae is the solution set in the other one, and vice versa. Consequently, if $G \neq \varnothing$ is another closed convex set with associated weak dual cone $M \leq$, we have

$$
F \subset G \Leftrightarrow M^{\leq} \subset K^{\leq}
$$

i.e., the containment of closed convex sets is actually reduced to checking the consistency of the given representation of $F$ and, if $F \neq \varnothing$, the calculus of the respective weak dual cones. Then the dual characterization of the containment is the right hand side inclusion in (3.4).

For Case 4, the following existence theorem allows to check the nonemptyness of $F$. Such result can be seen as a convex counterpart of the existence theorem of Zhu [13] for linear systems in infinite dimensional spaces (see Lemma 4.1 in [5] for a semi-infinite version).

Proposition 3.1 Let $F=\left\{x \in \mathbb{R}^{n} \mid f_{t}(x) \leq 0, \forall t \in W\right\}$, where $f_{t}: \mathbb{R}^{n} \rightarrow$ $\mathbb{R} \cup\{+\infty\}$ is proper, convex and lsc for all $t \in T$. Then $F \neq \varnothing$ if and only if

$$
\left(\begin{array}{c}
0_{n} \\
-1
\end{array}\right) \notin \mathrm{cl} \text { coneco }\left[\bigcup_{t \in W} \text { epi } f_{t}^{*}\right] \text {. }
$$

Proof. For each $t \in W$, we consider the function $h_{t}:=\gamma_{t} f_{t}$, where

$$
\gamma_{t}:= \begin{cases}1, & f_{t}\left(0_{n}\right) \leq 0 \\ f_{t}\left(0_{n}\right)^{-1}, & f_{t}\left(0_{n}\right)>0\end{cases}
$$

Since the function $h:=\sup _{t \in W} h_{t}$ is proper, convex and lsc, according to Theorem 2.4.4 in [7], we have

$$
\operatorname{cl}\left(\mathbb{R}_{+} \text {epi } h^{*}\right)=\operatorname{cl} \text { coneco }\left[\bigcup_{t \in W} \text { epi } h_{t}^{*}\right]=\operatorname{cl} \text { coneco }\left[\bigcup_{t \in W} \text { epi } f_{t}^{*}\right],
$$

with $F=\left\{x \in \mathbb{R}^{n} \mid h(x) \leq 0\right\}$. The conclusion follows from Proposition 2.2(i).

The nonhomogeneous Farkas Lemma for linear semi-infinite systems (Corollary 3.1.2 in [5], Corollary 3.3 in [8]) establishes that, if $F=\left\{x \in \mathbb{R}^{n} \mid a_{t}^{\prime} x \leq b_{t}, \forall t \in W\right\}$, then

$$
K^{\leq}=\mathrm{cl} \text { coneco }\left\{\left(\begin{array}{l}
a_{t} \\
b_{t}
\end{array}\right), t \in W ;\left(\begin{array}{c}
0_{n} \\
1
\end{array}\right)\right\} .
$$


Proposition 3.2 Let $F=\bigcap_{i \in I} F_{i} \neq \varnothing$, where $F_{i}$ is a closed convex set with weak duality cone $K_{i}^{\leq}, i \in I$. Then

$$
K^{\leq}=\operatorname{clco}\left[\bigcup_{i \in I} K_{i}^{\leq}\right] .
$$

Proof. Since $F_{i}=\left\{x \in \mathbb{R}^{n} \mid a^{\prime} x \leq b, \quad \forall\left(\begin{array}{l}a \\ b\end{array}\right) \in K_{i}^{\leq}\right\}$for all $i \in I$,

$$
F=\left\{x \in \mathbb{R}^{n} \mid a^{\prime} x \leq b, \quad \forall\left(\begin{array}{l}
a \\
b
\end{array}\right) \in \bigcup_{i \in I} K_{i}^{\leq}\right\} .
$$

Then, by the nonhomogeneous Farkas Lemma and since $\left(\begin{array}{c}0_{n} \\ 1\end{array}\right) \in K_{i}^{\leq}$for all $i \in I$, we have

$$
K^{\leq}=\operatorname{cl} \text { coneco }\left[\left(\bigcup_{i \in I} K_{i}^{\leq}\right) \cup\left\{\left(\begin{array}{c}
0_{n} \\
1
\end{array}\right)\right\}\right]=\operatorname{cl} \operatorname{co}\left[\bigcup_{i \in I} K_{i}^{\leq}\right] .
$$

Proposition 3.3 If $F=\left\{x \in \mathbb{R}^{n} \mid f_{t}(x) \leq 0, \forall t \in W\right\} \neq \varnothing$ and $f_{t}: \mathbb{R}^{n} \rightarrow$ $\mathbb{R} \cup\{+\infty\}$ is proper, convex and lsc for each $t \in W$, then the weak duality cone of $F$ is

$$
K^{\leq}=\operatorname{cl} \text { coneco }\left[\bigcup_{t \in T} \operatorname{epi} f_{t}^{*}\right] .
$$

Proof. $F=\bigcap_{t \in W} F_{t} \neq \varnothing$, with $F_{t}:=\left\{x \in \mathbb{R}^{n} \mid f_{t}(x) \leq 0\right\}$ for all $t \in W$. Then, by Propositions 3.2 and 2.2(ii),

$$
\begin{aligned}
K^{\leq} & =\mathrm{cl} \text { со }\left[\bigcup_{t \in W} K_{t}^{\leq}\right]=\mathrm{cl} \text { со }\left[\bigcup_{t \in W} \operatorname{cl}\left(\mathbb{R}_{+} \text {epi } f_{t}^{*}\right)\right] \\
& =\operatorname{cl} \text { со }\left[\bigcup_{t \in W}\left(\mathbb{R}_{+} \text {epi } f_{t}^{*}\right)\right]=\mathrm{cl} \text { coneco }\left[\bigcup_{t \in W} \text { epi } f_{t}^{*}\right] .
\end{aligned}
$$

Observe that $K \leq$ is the same cone which yields the consistency test (3.5). Theorem 2.4.4 in [7] provides an alternative proof of Proposition 3.3 (see Theorem 3.2 in [8]).

\section{Stable Set Containments}

In this section we see how the inclusion $F \subset G$, where $F$ and $G$ are represented by means of linear inequality systems, is preserved under sufficiently small perturbations of the data. To formulate the problem, let $F$ and $G$ be the solution sets of the systems

$$
\xi=\left\{a_{t}^{\prime} x \leq b_{t}, t \in W\right\}
$$


and

$$
\eta=\left\{c_{i}^{\prime} x \leq d_{i}, i \in I\right\}
$$

We say that the containment $F \subset G$ is stable if it holds under arbitrary perturbations of the coefficients of $\xi$ and $\eta$, provided that these perturbations are sufficiently small. In order to define the size of a perturbation, consider the set, $\Theta_{\xi}$, of all linear systems with the same number of unknowns and constraints as $\xi$. So the elements of $\Theta_{\xi}$ are of the form

$$
\xi_{1}=\left\{\left(a_{t}^{1}\right)^{\prime} x \leq b_{t}^{1}, t \in W\right\}
$$

with $a^{1}: W \rightarrow \mathbb{R}^{n}$ and $b^{1}: W \rightarrow \mathbb{R}$. The size of the perturbation which yields $\xi_{1}$ from the nominal system $\xi$ is defined as

$$
\rho\left(\xi_{1}, \xi\right):=\sup _{t \in W}\left\|\left(\begin{array}{l}
a_{t}^{1} \\
b_{t}^{1}
\end{array}\right)-\left(\begin{array}{l}
a_{t} \\
b_{t}
\end{array}\right)\right\|_{\infty} .
$$

It is easy to see that $\rho$ defines a pseudometric on $\Theta_{\xi}$ (observe that it is possible that $\left.\rho\left(\xi_{1}, \xi\right)=+\infty\right)$. Similarly, the nominal system defining $G, \eta$, provides perturbed systems in a space of parameters, $\Theta_{\eta}$, and the size of the perturbation is also measured by means of the pseudometric of the uniform convergence. We denote by $F_{1}$ and $G_{1}$ the solution sets of $\xi_{1}$ and $\eta_{1}$.

Precisely, the containment $F \subset G$ is stable, if there exists a scalar $\delta>0$ such that $F_{1} \subset G_{1}$ if $\rho\left(\xi_{1}, \xi\right)<\delta$ and $\rho\left(\eta_{1}, \eta\right)<\delta$.

We shall prove that the stable containment is basically the containment of a closed convex set in an open convex set, a particular case of containment of evenly convex sets.

Recall that $\eta$ satisfies the strongly Slater (SS) condition if there exists $\bar{x} \in \mathbb{R}^{n}$ and $\varepsilon>0$ such that $c_{i}^{\prime} \bar{x} \leq d_{i}-\varepsilon$ for all $i \in I$, i.e., if the system $\left\{c_{i}^{\prime} x+x_{n+1} \leq d_{i}, i \in\right.$ $\left.I ;-x_{n+1}<0\right\}$, is consistent, i.e. (by Proposition 2.1)

$$
0_{n+2} \notin \mathrm{eco}\left[\left(\begin{array}{c}
0_{n} \\
-1 \\
0
\end{array}\right)+\mathbb{R}_{+}\left\{\left(\begin{array}{c}
c_{i} \\
1 \\
d_{i}
\end{array}\right), i \in I\right\} ;\left(\begin{array}{c}
0_{n+1} \\
1
\end{array}\right)\right]
$$

Proposition 4.1 Let $F \neq \varnothing$ and $G$ be the solution sets of the linear system (4.1) and (4.2), respectively. Then the following statements hold:

(i) If $F \subset \operatorname{int} G, G$ is compact and $\eta$ satisfies the $S S$ condition, then $F \subset G$ is stable.

(ii) If $F \subset G$ is stable and either $\left\{a_{t}, t \in T\right\}$ or $\left\{c_{i}, i \in I\right\}$ is bounded (e.g., one of the two systems is ordinary), then $F \subset \operatorname{int} G$.

Proof. (i) Assume that $F \subset$ int $G, G$ is compact and $\eta$ satisfies the SS condition. Let $\varepsilon:=d(F, \operatorname{bd} G)>0$ ( $F$ is compact), $U:=\left\{x \in \mathbb{R}^{n} \mid d(x, F)<\frac{\varepsilon}{2}\right\}$, and $V:=\mathbb{R}^{n} \backslash \operatorname{cl} U$. Obviously, $U$ and $V$ are disjoint open sets such that $F \subset U$ and bd $G \subset V$, respectively. 
Since $F$ is bounded, the feasible set mapping associating to each $\xi_{1} \in \Theta_{\xi}$ its corresponding solution set mapping $F_{1}$ is Berge upper semicontinuous (Corollary 6.2.1 in [5]). Hence, there exists $\delta_{0}>0$ such that $F_{1} \subset U$ if $\rho\left(\xi_{1}, \xi\right)<\delta_{0}$.

The assumptions on $G$ and $\eta$ entail two consequences:

(a) There exists $\delta_{1}>0$ such that $G_{1} \cap U \neq \varnothing$ if $\rho\left(\eta_{1}, \eta\right)<\delta_{1}$ (the SS condition of $\eta$ is equivalent to the Berge lower semicontinuity of the feasible set mapping associating to each $\eta_{1} \in \Theta_{\eta}$ its solution set $G_{1}$, see e.g. Theorem 6.1 in [5]).

(b) There exists $\delta_{2}>0$ such that bd $G_{1} \subset V$ if $\rho\left(\eta_{1}, \eta\right)<\delta_{2}$ (since $G$ is a convex body and $\eta$ satisfies the SS condition, the set valued mapping associating to each $\eta_{1} \in \Theta_{\eta}$ the boundary of its solution set, bd $G_{1}$, is Berge upper semicontinuous, according to Corollary 5.3 in [6]).

Let $\delta=\min \left\{\delta_{0}, \delta_{1}, \delta_{2}\right\}>0$ and let $\xi_{1} \in \Theta_{\xi}$ and $\eta_{1} \in \Theta_{\eta}$ such that $\rho\left(\xi_{1}, \xi\right)<\delta$ and $\rho\left(\eta_{1}, \eta\right)<\delta$.

If $U \not \subset G_{1}$, we take $x^{1} \in U \backslash G_{1}$ and $x^{2} \in U \cap G_{1}$ (from (a)), and $\left[x^{1}, x^{2}\right]$ must contain a point $x^{3} \in\left[x^{1}, x^{2}\right] \subset U$ such that $x^{3} \in \operatorname{bd} G_{1}$. Then $x^{3} \in U \cap\left(\operatorname{bd} G_{1}\right) \subset$ $U \cap V$, by (b), contradicting $U \cap V=\varnothing$.

Therefore we have $F_{1} \subset U \subset G_{1}$.

(ii) Now we assume that $F \subset G$ but $F \not \subset$ int $G$. Let $\bar{x} \in F \backslash(\operatorname{int} G)$.

We shall prove that the inclusion $F \subset G$ is unstable provided that one of the two sets of left-hand-side vectors is bounded.

First we assume that $\left\{a_{t}, t \in T\right\}$ is bounded.

Since $\operatorname{int} G$ is evenly convex and $\bar{x} \notin \operatorname{int} G$, there exists $z \in \mathbb{R}^{n}$ such that $z^{\prime}(x-\bar{x})<0$ for all $x \in \operatorname{int} G$. Thus,

$$
z^{\prime}(x-\bar{x}) \leq 0 \text { for all } x \in G .
$$

Given $\gamma>0$, we consider the system $\xi_{\gamma}=\left\{a_{t}{ }^{\prime} x \leq b_{t}+\gamma a_{t}{ }^{\prime} z, t \in W\right\} \in \Theta_{\xi}$. Since the feasible set of $\xi_{\gamma}$ is $F_{\gamma}=F+\gamma z$, we have $\bar{x}+\gamma z \in F_{\gamma}$. On the other hand,

$$
z^{\prime}[(\bar{x}+\gamma z)-\bar{x}]=\gamma\|z\|^{2}>0,
$$

so that $\bar{x}+\gamma z \notin G$ according to (4.3). Hence $F_{\gamma} \not \subset G$, with

$$
\lim _{\gamma \searrow 0} \rho\left(\xi_{\gamma}, \xi\right)=\lim _{\gamma \searrow 0} \gamma\|z\| \sup _{t \in W}\left\|a_{t}\right\|=0 .
$$

Now we assume that $\left\{c_{i}, i \in I\right\}$ is bounded.

By the separation theorem (if $\bar{x} \notin G$ ) and the supporting hyperplane theorem (if $\bar{x} \in \operatorname{bd} G$ ), there exists $z \in \mathbb{R}^{n}, z \neq 0_{n}$, such that

$$
z^{\prime}(x-\bar{x}) \leq 0 \text { for all } x \in G .
$$


Given $\gamma>0$, we consider the system $\eta_{\gamma}=\left\{c_{i}^{\prime} x \leq d_{i}-\gamma c_{i}^{\prime} z, i \in I\right\}$. Now we have $G_{\gamma}=G-\gamma z$. If $\bar{x} \in G_{\gamma}$, then $\bar{x}+\gamma z \in G$ and (4.4) entails the following contradiction:

$$
0 \geq z^{\prime}[(\bar{x}+\gamma z)-\bar{x}]=\gamma\|z\|^{2}>0 .
$$

Since $\bar{x} \in F \backslash G_{\gamma}$, we have $F \not \subset G_{\gamma}$, with

$$
\lim _{\gamma \searrow 0} \rho\left(\eta_{\gamma}, \eta\right)=\lim _{\gamma \searrow 0} \gamma\|z\| \sup _{i \in I}\left\|c_{i}\right\|=0
$$

This completes the proof.

In particular, if $\eta$ is a minimal representation of a full dimensional polytope $G$, then

$$
F \subset G \text { is stable } \Leftrightarrow F \subset \operatorname{int} G,
$$

and the characterization of stable containment between closed convex sets is equivalent to the characterization of the containment of a closed convex set in an open convex set (the kind of problem we shall consider in the next section). This statement is not necessarily true for polyhedral sets (consider $n=2, G=\left\{x \in \mathbb{R}^{2} \mid x_{2} \leq 0\right\}$ and $F=\left\{x \in \mathbb{R}^{2} \mid x_{2} \leq-1\right\}$ ).

\section{The Containments of Evenly Convex Sets}

We define the strict dual cone of a nonempty evenly convex set $F \subset \mathbb{R}^{n}$ as

$$
K^{<}:=\left\{\left(\begin{array}{l}
a \\
b
\end{array}\right) \in \mathbb{R}^{n+1} \mid a^{\prime} x<b, \forall x \in F\right\} .
$$

Obviously, $\left\{0_{n}\right\} \times \mathbb{R}_{++} \subset K^{<}$and the equality holds if and only if $F=\mathbb{R}^{n}$. Since $0_{n+1} \notin K^{<}, K^{<}$cannot be closed. In particular, if $F$ is closed, we have $K^{<}$strictly contained in $K^{\leq}$as far as $0_{n+1} \in K^{\leq} \backslash K^{<}$(the supporting halfspaces for $F$ also define elements of $K \leq \backslash K^{<}$, if $\left.F \neq \mathbb{R}^{n}\right)$.

The symmetric expression of (5.1) is now a straightforward consequence of the characterization of the evenly convex sets by means of the strong separation property from external points:

$$
F=\left\{x \in \mathbb{R}^{n} \mid a^{\prime} x<b, \quad \forall\left(\begin{array}{l}
a \\
b
\end{array}\right) \in K^{<}\right\} .
$$

As for closed convex sets, if $M^{<}$denotes the strict dual cone of a second evenly convex set $G$,

$$
F \subset G \Leftrightarrow M^{<} \subset K^{<}
$$

(5.3) reduces again the containment problem with evenly convex sets to checking the consistency of the given representations of $F$ and $G$ (by means of Proposition 
2.1 if the given representation is linear and by means of the existence theorems in Section 6 otherwise) and, if $F \neq \varnothing \neq G$, the comparison of the respective strict dual cones. Their calculus is the main objective of this section. In particular cases $K^{<}$can be calculated through the weak dual cone of $\mathrm{cl} F, \bar{K}^{\leq}$. Obviously, $\bar{K}^{\leq}$is a closed convex cone and $\bar{K} \leq=K \leq$ if $F$ is closed.

From now on in this section we assume that $F$ is a nonempty evenly convex set with associated strict dual cone $K^{<}$. It follows from the definitions that $\mathrm{cl} K^{<}=\bar{K}^{\leq}$.

Thus the relative interiors of $\bar{K}^{\leq}$and $K^{<}$coincide and $K^{<}=\operatorname{int} \bar{K}^{\leq}$if $K^{<}$is open.

Observe also that $K^{<}$provides useful information on $F$, for instance, the recession cone of $F$ is

$$
0^{+} F=\left\{y \in \mathbb{R}^{n} \mid a^{\prime} y \leq 0, \forall\left(\begin{array}{l}
a \\
b
\end{array}\right) \in K^{<}\right\}
$$

(from (5.2)), and $F$ is bounded if and only if $\left(\begin{array}{c}0_{n} \\ 1\end{array}\right) \in \operatorname{int} K^{<}$.

Example 5.1 If $F=\mathbb{R}_{+}^{2}$, then $\bar{K}^{\leq}=K^{\leq}=\mathbb{R}_{-}^{2} \times \mathbb{R}_{+}$, where $\mathbb{R}_{-}:=-\mathbb{R}_{+}$, and $K^{<}=\mathbb{R}_{-}^{2} \times \mathbb{R}_{++}$. Observe that $K^{<}$is neither closed nor open, with $\mathrm{cl} K^{<}=\bar{K}^{\leq}$.

The next three results show the existence of a topological duality between the evenly convex sets and their respective strict dual cones. These relationships will allow us to calculate $K^{<}$from $\bar{K}^{\leq}$when $F$ is either open or compact.

Proposition 5.1 The cone $K^{<} \cup\left\{0_{n+1}\right\}$ is closed if and only if the set $F$ is open. In such a case, $K^{<}=\bar{K}^{\leq} \backslash\left\{0_{n+1}\right\}$.

Proof. Assume that $F$ is not open. Let $\bar{x} \in(\operatorname{bd} F) \cap F$ and let $a \neq 0_{n}$ and $b \in \mathbb{R}$ such that $a^{\prime} x \leq b$ for all $x \in F$ and $a^{\prime} \bar{x}=b\left(a^{\prime} x=b\right.$ is a supporting hyperplane for $F$ at $\bar{x})$. Then, clearly, $\left(\begin{array}{l}a \\ b\end{array}\right) \in \operatorname{cl}\left(K^{<} \cup\left\{0_{n+1}\right\}\right)$. On the other hand, $a^{\prime} \bar{x}=b$, with $\bar{x} \in F$, entails $\left(\begin{array}{l}a \\ b\end{array}\right) \notin K^{<}$. Since $a \neq 0_{n},\left(\begin{array}{l}a \\ b\end{array}\right) \notin K^{<} \cup\left\{0_{n+1}\right\}$ and we conclude that this set is not closed.

Now we assume that $K^{<} \cup\left\{0_{n+1}\right\}$ is not closed. Let

$$
\left\{\left(\begin{array}{l}
a_{r} \\
b_{r}
\end{array}\right)\right\} \subset K^{<} \cup\left\{0_{n+1}\right\}
$$

such that

$$
\lim _{r}\left(\begin{array}{l}
a_{r} \\
b_{r}
\end{array}\right)=\left(\begin{array}{l}
a \\
b
\end{array}\right) \notin K^{<} \cup\left\{0_{n+1}\right\} .
$$

Let $\bar{x} \in F$ such that $a^{\prime} \bar{x} \geq b$. Since $a_{r}^{\prime} \bar{x}<b_{r}$ for all $r \in \mathbb{N}$, we have, for $r \rightarrow$ $\infty, a^{\prime} \bar{x} \leq b$, i.e., $a^{\prime} \bar{x}=b$. We shall prove that $\bar{x} \in(\mathrm{bd} F) \cap F$, so that $F$ cannot be open. 
We have $a \neq 0_{n}$ (otherwise $\left(\begin{array}{l}a \\ b\end{array}\right)=0_{n+1} \in K^{<} \cup\left\{0_{n+1}\right\}$ ), and $a^{\prime} x=b$ defines a hyperplane containing $\bar{x}$. Since $\left(\begin{array}{l}a_{r} \\ b_{r}\end{array}\right) \in K^{<} \cup\left\{0_{n+1}\right\}$ for all $r \in \mathbb{N}$, either we have $a_{r}^{\prime} x=b_{r}$ for all $x \in F$ (if $\left(\begin{array}{l}a_{r} \\ b_{r}\end{array}\right)=0_{n+1}$ ) or $a_{r}^{\prime} x<b_{r}$ for all $x \in F$, otherwise. Taking limits, $a^{\prime} x \leq b$ for all $x \in F$ and so $a^{\prime} x=b$ is a supporting hyperplane for $F$ at $\bar{x}$. Hence $\bar{x} \in(\operatorname{bd} F) \cap F$.

If $K^{<} \cup\left\{0_{n+1}\right\}$ is closed, then $K^{<} \cup\left\{0_{n+1}\right\}=\bar{K} \leq$ and, since $0_{n+1} \notin K^{<}$, $K^{<}=\bar{K} \leq \backslash\left\{0_{n+1}\right\}$.

From Proposition 5.1 we get a particular version of Farkas Lemma for linear strict inequalities.

Corollary 5.1 If $|S|<\infty, a^{\prime} x<b$ is a consequence of the consistent system $\left\{a_{t}^{\prime} x<\right.$ $\left.b_{t}, t \in S\right\}$ if and only if

$$
\left(\begin{array}{l}
a \\
b
\end{array}\right) \in\left[\operatorname{coneco}\left\{\left(\begin{array}{l}
a_{t} \\
b_{t}
\end{array}\right), t \in S ;\left(\begin{array}{l}
0 \\
1
\end{array}\right)\right\}\right] \backslash\left\{0_{n+1}\right\} .
$$

Proof. The assumptions guarantee that $F:=\left\{x \in \mathbb{R}^{n} \mid a_{t}^{\prime} x<b_{t}, t \in S\right\}$ is an open subset of $\mathbb{R}^{n}$. The weak dual cone of $\mathrm{cl} F$ is, by the nonhomegeneous Farkas Lemma, the polyhedral convex cone

$$
\bar{K}^{\leq}=\text {coneco }\left\{\left(\begin{array}{c}
a_{t} \\
b_{t}
\end{array}\right), t \in S ;\left(\begin{array}{c}
0_{n} \\
1
\end{array}\right)\right\},
$$

and the conclusion follows from Proposition 5.1.

Proposition 5.2 If the cone $K^{<}$is relatively open, then the set $F$ is closed.

Proof. Assume that $F$ is not closed. Let $\left\{x_{r}\right\} \subset F$ such that $\lim _{r} x_{r}=\bar{x} \notin F$. Since $F$ is an evenly convex set, there exists $a \neq 0_{n}$ and $b \in \mathbb{R}$ such that $a^{\prime} x<b$ for all $x \in F$ and $a^{\prime} \bar{x}=b$. Obviously, $\left(\begin{array}{l}a \\ b\end{array}\right) \in K^{<}$.

Take an arbitrary small $\varepsilon>0$. Since $\lim _{r} a^{\prime} x_{r}=a^{\prime} \bar{x}=b>b-\varepsilon$, there exists $m \in$ $\mathbb{N}$ such that $a^{\prime} x_{m}>b-\varepsilon$, with $x_{m} \in F$. Then $\left(\begin{array}{c}a \\ b-\varepsilon\end{array}\right) \notin K^{<}$, with $\left\|\left(\begin{array}{c}a \\ b-\varepsilon\end{array}\right)-\left(\begin{array}{l}a \\ b\end{array}\right)\right\|=$ $\varepsilon$ and $\left(\begin{array}{c}a \\ b-\varepsilon\end{array}\right)=\left(\begin{array}{l}a \\ b\end{array}\right)-\varepsilon\left(\begin{array}{c}0_{n} \\ 1\end{array}\right) \in$ aff $K^{<}$. Hence $\left(\begin{array}{l}a \\ b\end{array}\right)$ does not belong to the relative interior of $K^{<}$.

Example 5.1 shows that the converse of Proposition 5.2 is not true. Next we show that the compactness of $F$ guarantees the openness (not only relative) of $K^{<}$.

Proposition 5.3 If $F$ is compact, then $K^{<}$is open. In such a case, $K^{<}=\operatorname{int} \bar{K}^{\leq}$. 
Proof. Assume that $F$ is compact. Then its support function $\sigma_{F}$ is continuous on $\mathbb{R}^{n}$.

Let $\left(\begin{array}{l}a \\ b\end{array}\right) \in K^{<}$. Since $\sigma_{F}(a)=\max _{x \in F} a^{\prime} x<b$, there exists $\varepsilon>0$ such that $\sigma_{F}(a)<b-\varepsilon$. Let $\delta>0$ such that $\delta<\frac{\varepsilon}{2}$ and

$$
\left|\sigma_{F}(c)-\sigma_{F}(a)\right|<\frac{\varepsilon}{2} \quad \text { if }\|c-a\|<\delta .
$$

We shall prove that $K^{<}$contains the open ball, in $\mathbb{R}^{n+1}$, centered at $\left(\begin{array}{l}a \\ b\end{array}\right)$ with radius $\delta$. In fact, if $\left\|\left(\begin{array}{l}c \\ d\end{array}\right)-\left(\begin{array}{l}a \\ b\end{array}\right)\right\|<\delta$, then

$$
\sigma_{F}(c)<\sigma_{F}(a)+\frac{\varepsilon}{2}<b-\frac{\varepsilon}{2}<d .
$$

Thus $\max _{x \in F} c^{\prime} x<d$, i.e., $\left(\begin{array}{l}c \\ d\end{array}\right) \in K^{<}$.

Proposition 5.3 yields another version of Farkas Lemma for linear strict inequalities.

Corollary 5.2 If the solution set of $\left\{a_{t}^{\prime} x<b_{t}, t \in S\right\}$ is compact, then $a^{\prime} x<b$ is a consequence of that system if and only if

$$
\left(\begin{array}{l}
a \\
b
\end{array}\right) \in \operatorname{int} \text { coneco }\left\{\left(\begin{array}{l}
a_{t} \\
b_{t}
\end{array}\right), t \in S ;\left(\begin{array}{c}
0_{n} \\
1
\end{array}\right)\right\} .
$$

Proof. Since $F:=\left\{x \in \mathbb{R}^{n} \mid a_{t}^{\prime} x<b_{t}, \forall t \in S\right\}$ is compact, by Proposition 5.3,

$$
\begin{aligned}
K^{<} & =\text {int cl coneco }\left\{\left(\begin{array}{l}
a_{t} \\
b_{t}
\end{array}\right), t \in S ;\left(\begin{array}{c}
0_{n} \\
1
\end{array}\right)\right\} \\
& =\text { int coneco }\left\{\left(\begin{array}{c}
a_{t} \\
b_{t}
\end{array}\right), t \in S ;\left(\begin{array}{c}
0_{n} \\
1
\end{array}\right)\right\} .
\end{aligned}
$$

Example 5.2 It is easy to see that the unit closed disk $F:=\left\{x \in \mathbb{R}^{2} \mid\|x\| \leq 1\right\}$ is the solution set of the system of strict inequalities

$$
\left\{(\cos t) x_{1}+(\sin t) x_{2}<s,(t, s) \in[0,2 \pi] \times\right] 1,+\infty[\} .
$$

Since $F=\left\{x \in \mathbb{R}^{2} \mid(\cos t) x_{1}+(\sin t) x_{2} \leq 1, t \in[0,2 \pi]\right\}$, we have

$$
\begin{aligned}
\bar{K}^{\leq} & =\operatorname{coneco}\left\{\left(\begin{array}{c}
\cos t \\
\sin t \\
1
\end{array}\right), t \in[0,2 \pi] ;\left(\begin{array}{c}
0_{2} \\
1
\end{array}\right)\right\} \\
& =\left\{x \in \mathbb{R}^{3} \mid x_{1}^{2}+x_{2}^{2} \leq 1, x_{3} \geq 0\right\} .
\end{aligned}
$$

Thus,

$$
K^{<}=\operatorname{int} \bar{K}^{\leq}=\left\{x \in \mathbb{R}^{3} \mid x_{1}^{2}+x_{2}^{2}<1, x_{3}>0\right\} .
$$


The next result can be interpreted as the general Farkas Lemma for systems of strict inequalities.

Proposition 5.4 If $F=\left\{x \in \mathbb{R}^{n} \mid a_{t}^{\prime} x<b_{t}, \forall t \in S\right\}$, then

$$
K^{<}=\operatorname{eco} \mathbb{R}_{++}\left\{\left(\begin{array}{c}
a_{t} \\
b_{t}
\end{array}\right), t \in S ;\left(\begin{array}{c}
0_{n} \\
1
\end{array}\right)\right\} .
$$

Proof. We have to prove that $K^{<}=\operatorname{eco} X$, where $X$ is the cone

$$
X:=\mathbb{R}_{++}\left\{\left(\begin{array}{c}
a_{t} \\
b_{t}
\end{array}\right), t \in S ;\left(\begin{array}{c}
0_{n} \\
1
\end{array}\right)\right\} .
$$

Assume that $\left(\begin{array}{l}a \\ b\end{array}\right) \notin \operatorname{eco} X$. Then either $\left(\begin{array}{l}a \\ b\end{array}\right) \notin \operatorname{cl} \operatorname{co} X$ or $\left(\begin{array}{l}a \\ b\end{array}\right)$ belongs to a certain exposed face of cl co $X$ which does not contain points of $X$, otherwise. In both cases, there exists a hyperplane containing $\left(\begin{array}{l}a \\ b\end{array}\right)$ and $0_{n+1}$ which does not contain points of $X$.

Let $\left(\begin{array}{l}c \\ d\end{array}\right) \neq 0_{n+1}$ such that

$$
\left(\begin{array}{l}
c \\
d
\end{array}\right)^{\prime}\left(\begin{array}{l}
a \\
b
\end{array}\right)=0 \text { and }\left(\begin{array}{l}
c \\
d
\end{array}\right)^{\prime}\left(\begin{array}{l}
v \\
w
\end{array}\right)<0 \text { for all }\left(\begin{array}{c}
v \\
w
\end{array}\right) \in X .
$$

From (5.4), since $\left(\begin{array}{c}0_{n} \\ 1\end{array}\right) \in X$, we get $d<0$. Let $\bar{x}:=|d|^{-1} c$. Multiplying by $|d|^{-1}$ each expression of (5.4), we obtain $a^{\prime} \bar{x}=b$ and $\left(\begin{array}{c}\bar{x} \\ -1\end{array}\right)^{\prime}\left(\begin{array}{c}v \\ w\end{array}\right)<0$ for all $\left(\begin{array}{c}v \\ w\end{array}\right) \in X$. In particular, since $\left(\begin{array}{l}a_{t} \\ b_{t}\end{array}\right) \in X$ if $t \in S$, we get $a_{t}^{\prime} \bar{x}<b_{t}$ for all $t \in S$. Therefore $\bar{x} \in F$ and $a^{\prime} \bar{x}=b$, and this entails $\left(\begin{array}{l}a \\ b\end{array}\right) \notin K^{<}$.

Now we assume $\left(\begin{array}{l}a \\ b\end{array}\right) \in \operatorname{eco} X$. Since

$$
\text { eco } X \subset \operatorname{clco} X \subset \operatorname{cl} \text { coneco }\left\{\left(\begin{array}{l}
a_{t} \\
b_{t}
\end{array}\right), t \in S ;\left(\begin{array}{c}
0_{n} \\
1
\end{array}\right)\right\}=\bar{K}^{\leq},
$$

we have $a^{\prime} x \leq b$ for all $x \in F$. We claim that $\left(\begin{array}{l}a \\ b\end{array}\right) \in K^{<}$. Indeed, if there exists $\bar{x} \in F$ such that $a^{\prime} \bar{x}=b$ then

$$
\left(\begin{array}{c}
\bar{x} \\
-1
\end{array}\right)^{\prime}\left[\lambda\left(\begin{array}{l}
a_{t} \\
b_{t}
\end{array}\right)\right]=\lambda\left(a_{t}^{\prime} \bar{x}-b_{t}\right)<0, \quad \forall \lambda>0, \forall t \in S,
$$


and

$$
\left(\begin{array}{c}
\bar{x} \\
-1
\end{array}\right)^{\prime}\left[\lambda\left(\begin{array}{c}
0_{n} \\
1
\end{array}\right)\right]=-\lambda<0, \quad \forall \lambda>0
$$

It follows from (5.5) and (5.6) that $\left(\begin{array}{c}\bar{x} \\ -1\end{array}\right)^{\prime}\left(\begin{array}{c}v \\ w\end{array}\right)<0$ for all $\left(\begin{array}{c}v \\ w\end{array}\right) \in X$ and hence,

$$
\left(\begin{array}{c}
\bar{x} \\
-1
\end{array}\right)^{\prime}\left[\left(\begin{array}{c}
v \\
w
\end{array}\right)-\left(\begin{array}{l}
a \\
b
\end{array}\right)\right]<0 \text { for all }\left(\begin{array}{c}
v \\
w
\end{array}\right) \in X
$$

as $\left(\begin{array}{c}\bar{x} \\ -1\end{array}\right)^{\prime}\left(\begin{array}{l}a \\ b\end{array}\right)=0$. Recalling the last characterization of eco $X$ in the preliminaries, (5.7) entails $\left(\begin{array}{l}a \\ b\end{array}\right) \notin \operatorname{eco} X$. This contradiction proves that $\left(\begin{array}{l}a \\ b\end{array}\right) \in K^{<}$.

Observe that $\left\{a_{t}^{\prime} x<b_{t}, t \in S\right\}$ is consistent if and only if

$$
\left\{\left(\lambda a_{t}\right)^{\prime} x<\lambda b_{t},(\lambda, t) \in \mathbb{R}_{++} \times S ;\left(\mu 0_{n}\right)^{\prime} x<\mu, \mu \in \mathbb{R}_{++}\right\}
$$

is consistent if and only if (by Proposition 2.1)

$$
\begin{gathered}
0_{n+1} \notin \text { eco }\left[\mathbb{R}_{++}\left\{\left(\begin{array}{c}
a_{t} \\
b_{t}
\end{array}\right), t \in S ;\left(\begin{array}{c}
0_{n} \\
1
\end{array}\right)\right\} ;\left(\begin{array}{c}
0_{n} \\
1
\end{array}\right)\right] \\
=\operatorname{eco} \mathbb{R}_{++}\left\{\left(\begin{array}{c}
a_{t} \\
b_{t}
\end{array}\right), t \in S ;\left(\begin{array}{c}
0_{n} \\
1
\end{array}\right)\right\} .
\end{gathered}
$$

Thus $K^{<}$(defined as in Proposition 5.4) characterizes the consistency of $\left\{a_{t}^{\prime} x<\right.$ $\left.b_{t}, t \in S\right\}$ by $0_{n+1} \notin K^{<}$, in the same way as $\left(\begin{array}{c}0_{n} \\ -1\end{array}\right) \notin K^{\leq}$(defined in (3.6)) characterizes the consistency of $\left\{a_{t}^{\prime} x \leq b_{t}, t \in W\right\}$.

The next result is the counterpart of Proposition 3.2 for strict dual cones.

Proposition 5.5 Let $F=\bigcap_{i \in I} F_{i} \neq \varnothing$, where $F_{i}$ is an evenly convex set with strict dual cone $K_{i}^{<}, i \in I$. Then

$$
K^{<}=\operatorname{eco}\left[\bigcup_{i \in I} K_{i}^{<}\right]
$$

Proof. By (5.2), $F_{i}=\left\{x \in \mathbb{R}^{n} \mid a^{\prime} x<b, \forall\left(\begin{array}{l}a \\ b\end{array}\right) \in K_{i}^{<}\right\}$for all $i \in I$. Then

$$
F=\left\{x \in \mathbb{R}^{n} \mid a^{\prime} x<b, \forall\left(\begin{array}{l}
a \\
b
\end{array}\right) \in \bigcup_{i \in I} K_{i}^{<}\right\}
$$


and, recalling Proposition 5.4 and that $\left(\begin{array}{c}0_{n} \\ 1\end{array}\right) \in K_{i}^{<}$for all $i \in I$, we have

$$
K^{<}=\operatorname{eco} \mathbb{R}_{++}\left[\bigcup_{i \in I} K_{i}^{<}\right]=\operatorname{eco}\left[\bigcup_{i \in I} K_{i}^{<}\right] \text {. }
$$

Now we can calculate the strict dual cone of certain evenly convex sets.

Corollary 5.3 Let $F=\left\{x \in \mathbb{R}^{n} \mid f_{t}(x)<0, \forall t \in S\right\} \neq \varnothing$, with $f_{t}: \mathbb{R}^{n} \rightarrow \mathbb{R}$ convex for all $t \in S$. Then the strict dual cone of $F$ is

$$
K^{<}=\operatorname{eco}\left[\left\{\bigcup_{t \in S} \operatorname{cl}\left(\mathbb{R}_{+} \text {epi } f_{t}^{*}\right)\right\} \backslash\left\{0_{n+1}\right\}\right] \text {. }
$$

In the particular case of $f_{t}(x)=a_{t}^{\prime} x-b_{t}$ for all $t \in S$ (i.e., $F$ is the solution set of a system of linear strict inequalities), it holds

$$
K^{<}=\operatorname{eco}\left[\left(\operatorname{coneco}\left\{\left(\begin{array}{l}
a_{t} \\
b_{t}
\end{array}\right), t \in S ;\left(\begin{array}{c}
0_{n} \\
1
\end{array}\right)\right\}\right) \backslash\left\{0_{n+1}\right\}\right] \text {. }
$$

Proof. Given $t \in S$, we denote $F_{t}:=\left\{x \in \mathbb{R}^{n} \mid f_{t}(x)<0\right\}$, which is a nonempty open convex set. The weak dual cone of

$$
\operatorname{cl} F_{t}=\left\{x \in \mathbb{R}^{n} \mid f_{t}(x) \leq 0\right\}
$$

is, according to Proposition 2.2,

$$
\bar{K}_{t}^{\leq}=\operatorname{cl}\left(\mathbb{R}_{+} \text {epi } f_{t}^{*}\right) .
$$

Then, recalling Proposition 5.1, the strict dual cone of $F_{t}$ is

$$
K_{t}^{<}=\left[\operatorname{cl}\left(\mathbb{R}_{+} \text {epi } f_{t}^{*}\right)\right] \backslash\left\{0_{n+1}\right\},
$$

and, by Proposition 5.5, we have

$$
K^{<}=\operatorname{eco}\left[\left\{\bigcup_{t \in S} \operatorname{cl}\left(\mathbb{R}_{+} \text {epi } f_{t}^{*}\right)\right\} \backslash\left\{0_{n+1}\right\}\right] .
$$

If $f_{t}(x)=a_{t}^{\prime} x-b_{t}$, then $f_{t}^{*}=b_{t}+\delta_{\left\{a_{t}\right\}}$ and

$$
\operatorname{cl}\left(\mathbb{R}_{+} \text {epi } f_{t}^{*}\right)=\operatorname{coneco}\left\{\left(\begin{array}{c}
a_{t} \\
b_{t}
\end{array}\right),\left(\begin{array}{c}
0_{n} \\
1
\end{array}\right)\right\}
$$

so that

$$
\begin{aligned}
K^{<} & =\operatorname{eco}\left[\left(\bigcup_{t \in S} \operatorname{coneco}\left\{\left(\begin{array}{c}
a_{t} \\
b_{t}
\end{array}\right),\left(\begin{array}{c}
0_{n} \\
1
\end{array}\right)\right\}\right) \backslash\left\{0_{n+1}\right\}\right] \\
& =\operatorname{eco}\left[\left(\operatorname{coneco}\left\{\left(\begin{array}{c}
a_{t} \\
b_{t}
\end{array}\right), t \in S ;\left(\begin{array}{c}
0_{n} \\
1
\end{array}\right)\right\}\right) \backslash\left\{0_{n+1}\right\}\right] .
\end{aligned}
$$


Example 5.1 (revisited) $F=\left\{x \in \mathbb{R}^{2} \mid-x_{1}<\lambda, \forall \lambda \in \mathbb{R}_{++} ;-x_{2}<\mu, \forall \mu \in\right.$ $\left.\mathbb{R}_{++}\right\}$. Since

$$
\text { coneco }\left\{\left(\begin{array}{c}
-1 \\
0 \\
\lambda
\end{array}\right), \lambda>0 ;\left(\begin{array}{c}
0 \\
-1 \\
\mu
\end{array}\right), \mu>0 ;\left(\begin{array}{l}
0 \\
0 \\
1
\end{array}\right)\right\} \backslash\left\{0_{3}\right\}=\mathbb{R}_{-}^{2} \times \mathbb{R}_{++},
$$

and this set is evenly convex, $K^{<}=\mathbb{R}_{-}^{2} \times \mathbb{R}_{++}$.

Corollary 5.4 Let $F=\left\{x \in \mathbb{R}^{n} \mid f_{t}(x) \leq 0, \forall t \in W\right\} \neq \varnothing$, with $f_{t}: \mathbb{R}^{n} \rightarrow$ $\mathbb{R} \cup\{+\infty\}$ proper convex lsc for all $t \in W$. Let $K_{t}^{<}$be the strict dual cone of $F_{t}:=\left\{x \in \mathbb{R}^{n} \mid f_{t}(x) \leq 0\right\}, t \in W$. Then

$$
K^{<}=\operatorname{eco}\left[\bigcup_{t \in W} K_{t}^{<}\right]
$$

where

$$
K_{t}^{<}= \begin{cases}\operatorname{int}\left(\mathbb{R}_{+} \text {epi } f_{t}^{*}\right), & \text { if } F_{t} \text { is bounded }, \\
\left(\operatorname{coneco}\left\{\left(\begin{array}{c}
a_{t} \\
b_{t}
\end{array}\right),\left(\begin{array}{c}
0_{n} \\
1
\end{array}\right)\right\}\right) \backslash \mathbb{R}_{+}\left\{\left(\begin{array}{c}
a_{t} \\
b_{t}
\end{array}\right)\right\}, & \text { if } f_{t}(x)=a_{t}^{\prime} x-b_{t} .\end{cases}
$$

Proof. It is a straightforward consequence of Propositions 5.3 (if $F_{t}$ is bounded) and 5.5, and the evenly convex property of $K_{t}^{<}$(if $f_{t}$ is an affine function).

Example 5.2 (revisited) $F=\left\{x \in \mathbb{R}^{2} \mid(\cos t) x_{2}+(\sin t) x_{t} \leq 1, \forall t \in[0,2 \pi]\right\}$.

By Corollary 5.4, recalling that eco $X=X$ if $X$ is open and convex,

$$
\begin{aligned}
K^{<}= & \operatorname{eco}\left\{\bigcup_{t \in[0,2 \pi]}\left[\left(\operatorname{coneco}\left\{\left(\begin{array}{c}
\cos t \\
\sin t \\
1
\end{array}\right),\left(\begin{array}{l}
0 \\
0 \\
1
\end{array}\right)\right\}\right) \backslash \mathbb{R}_{+}\left\{\left(\begin{array}{c}
\cos t \\
\sin t \\
1
\end{array}\right)\right\}\right]\right\} \\
& =\operatorname{eco}\left\{x \in \mathbb{R}^{3} \mid x_{1}^{2}+x_{2}^{2}<1, x_{3}>0\right\} \\
& =\left\{x \in \mathbb{R}^{3} \mid x_{1}^{2}+x_{2}^{2}<1, x_{3}>0\right\} .
\end{aligned}
$$

If $F=\left\{x \in \mathbb{R}^{n} \mid f_{t}(x)<0, \forall t \in S ; f_{t}(x) \leq 0, \forall t \in W\right\} \neq \varnothing$, its strict dual cone is $\operatorname{eco}\left(M^{<} \cup N^{<}\right)$, where $M^{<}$and $N^{<}$are the strict dual cones of

$$
\left\{x \in \mathbb{R}^{n} \mid f_{t}(x)<0, \forall t \in S\right\}
$$

and

$$
\left\{x \in \mathbb{R}^{n} \mid f_{t}(x) \leq 0, \forall t \in W\right\},
$$

respectively. $M^{<}$and $N^{<}$can be calculated by means of Corollaries 5.3 and 5.4. 


\section{General Existence Theorems and Applications to Set Containments}

This section provides three existence theorems for convex systems of the form

$$
\xi=\left\{f_{t}(x)<0, t \in S ; f_{t}(x) \leq 0, t \in W\right\},
$$

with $S \neq \varnothing$ (otherwise Proposition 3.1 applies). All the proposed consistency tests are expressed (or can be expressed by means of (2.1)) in terms of the information on $\left\{f_{t}, t \in S \cup W\right\}$, and the proofs will be derived from the existence theorem for linear systems (Proposition 2.1). The first result replaces linearity by sublinearity in Proposition 2.1.

Proposition 6.1 Let $f_{t}(x)=g_{t}(x)-b_{t}$, with $g_{t}: \mathbb{R}^{n} \rightarrow \mathbb{R}$ sublinear and $b_{t} \in \mathbb{R}$ for all $t \in S \cup W$. Then $\xi$ is consistent if and only if

$$
0_{n+1} \notin \operatorname{eco}\left[\left(\bigcup_{t \in S} \partial g_{t}\left(0_{n}\right) \times\left\{b_{t}\right\}\right)+\mathbb{R}_{+}\left(\bigcup_{t \in W} \partial g_{t}\left(0_{n}\right) \times\left\{b_{t}\right\}\right) ;\left(\begin{array}{c}
0_{n} \\
1
\end{array}\right)\right] \text {. }
$$

Proof. Since $g_{t}$ is sublinear and continuous, it can be expressed as

$$
g_{t}(x)=\max _{v \in \partial g_{t}\left(0_{n}\right)} v^{\prime} x \text { for all } x \in \mathbb{R}^{n} .
$$

Consequently, $\xi$ has the same solution set as the linear system

$$
\eta:=\left\{\begin{array}{c}
a_{t v}^{\prime} x<b_{t}, a_{t v} \in \partial g_{t}\left(0_{n}\right), t \in S \\
a_{t v}^{\prime} x \leq b_{t}, a_{t v} \in \partial g_{t}\left(0_{n}\right), t \in W
\end{array}\right\}
$$

Applying Proposition 2.1, we conclude that $\eta$ is consistent if and only if condition (6.1) holds.

It is easy to extend Proposition 6.1 to the case that each function $f_{t}$ can be expressed as $f_{t}(x)=g_{t}\left(x-x_{t}\right)-b_{t}$, with $g_{t}: \mathbb{R}^{n} \rightarrow \mathbb{R}$ sublinear, $x_{t} \in \mathbb{R}^{n}$ and $b_{t} \in \mathbb{R}$ (replace $x$ with $x-x_{t}$ and $0_{n}$ with $x_{t}$ in $\eta$ ). Typical examples of such functions are $f_{t}(x)=\sqrt{\left(x-x_{t}\right)^{\prime} A_{t}\left(x-x_{t}\right)}-b_{t}$ where $A_{t}$ is a positive definite symmetric matrix $x_{t} \in \mathbb{R}^{n}$ and $b_{t}>0$, so that the solution set of $\xi$ is the intersection of (open and closed) ellipsoids.

Next, we relax in another way the assumption on $\left\{f_{t}, t \in W\right\}$ in Proposition 6.1.

Proposition 6.2 Let $f_{t}: \mathbb{R}^{n} \rightarrow \mathbb{R} \cup\{+\infty\}$ proper convex lsc for all $t \in W$ and let $f_{t}(x)=g_{t}(x)-b_{t}$, with $g_{t}: \mathbb{R}^{n} \rightarrow \mathbb{R}$ sublinear for all $t \in S$. Then $\xi$ is consistent if and only if

$$
0_{n+1} \notin \operatorname{eco}\left[\left(\bigcup_{t \in S} \partial g_{t}\left(0_{n}\right) \times\left\{b_{t}\right\}\right)+\mathbb{R}_{+}\left\{\bigcup_{t \in W} \text { epi } f_{t}^{*}\right\} ;\left(\begin{array}{c}
0_{n} \\
1
\end{array}\right)\right] .
$$


Proof. Given $t \in S$, by (6.2), the solution set of $\left\{f_{t}(x)<0\right\}$ is the same as the solution set of

$$
\left\{a_{t v}^{\prime} x<b_{t}, a_{t v} \in \partial g_{t}\left(0_{n}\right)\right\} .
$$

On the other hand, given $t \in W$, we can write

$$
f_{t}(x)=f_{t}^{* *}(x)=\sup _{x^{*} \in \operatorname{dom} f_{t}^{*}}\left[x^{*}(x)-f_{t}^{*}\left(x^{*}\right)\right] \quad \text { for all } x \in \mathbb{R}^{n} .
$$

Thus $f_{t}(x) \leq 0$ if and only if $x^{*}(x)-f_{t}^{*}\left(x^{*}\right) \leq 0$ for all $x^{*} \in \operatorname{dom} f_{t}^{*}$ if and only if

$$
x^{*}(x) \leq f_{t}^{*}\left(x^{*}\right)+\gamma \text { for all }\left(x^{*}, \gamma\right) \in \operatorname{dom} f_{t}^{*} \times \mathbb{R}_{+} .
$$

Then $\xi$ is consistent if and only $\eta$ is consistent, where

$$
\eta:=\left\{\begin{array}{c}
a_{t v}^{\prime} x<b_{t}, a_{t v} \in \partial g_{t}\left(0_{n}\right), t \in S \\
x^{*}(x) \leq f_{t}^{*}\left(x^{*}\right)+\gamma,\left(x^{*}, \gamma\right) \in \operatorname{dom} f_{t}^{*} \times \mathbb{R}_{+}, t \in W
\end{array}\right\} .
$$

Applying again Proposition 2.1, we conclude that $\eta$ is consistent if and only if (6.3) holds.

The sublinearity assumption in Proposition 6.2 can be relaxed by requiring that each function $f_{t}, t \in S$, is the maximum of a family of affine functions (compare with (6.2)).

Proposition 6.3 Let $f_{t}: \mathbb{R}^{n} \rightarrow \mathbb{R} \cup\{+\infty\}$ proper convex lsc for all $t \in W$. We also assume that, for each $t \in S$, there exists a compact set $C_{t} \subset \mathbb{R}^{n+1}$ such that $f_{t}(x)=\max _{(a, b) \in C_{t}}(a x-b)$ for all $x \in \mathbb{R}^{n}$. Then $\xi$ is consistent if and only if

$$
0_{n+1} \notin \text { eco }\left[\left(\bigcup_{t \in S} C_{t}\right)+\mathbb{R}_{+}\left\{\bigcup_{t \in W} \operatorname{epi} f_{t}^{*}\right\} ;\left(\begin{array}{c}
0_{n} \\
1
\end{array}\right)\right] .
$$

Proof. Given $t \in S, f_{t}(x)<0$ if and only if

$$
\left(\begin{array}{l}
a \\
b
\end{array}\right)^{\prime}\left(\begin{array}{c}
x \\
-1
\end{array}\right)<0 \text { for all }\left(\begin{array}{l}
a \\
b
\end{array}\right) \in C_{t} .
$$

Reasoning as in Proposition 6.2, $\xi$ is consistent if and only if $\eta$ is consistent, with

$$
\eta:=\left\{\begin{array}{c}
a^{\prime} x<b,\left(\begin{array}{l}
a \\
b
\end{array}\right) \in C_{t}, t \in S \\
x^{*}(x) \leq f_{t}^{*}\left(x^{*}\right)+\gamma,\left(x^{*}, \gamma\right) \in \operatorname{dom} f_{t}^{*} \times \mathbb{R}_{+}, t \in W
\end{array}\right\},
$$

and $\eta$ turns out to be consistent if and only if (6.4) holds.

In order to summarize the consequences of the previous existence theorems for the containment problem, let us denote by $\mathcal{A}$ the set of affine functions on $\mathbb{R}^{n}$, by 
$\mathcal{S}$ the family of differences between sublinear (possibly composed with translations) and constant functions, by $\mathcal{M}$ the family of functions which can be expressed as $\max _{(a, b) \in C}(a x-b)$ for a certain compact set $C \subset \mathbb{R}^{n+1}$, and by $\mathcal{C}$ the class of proper convex lsc functions.

Given $F=\left\{x \in \mathbb{R}^{n} \mid f_{t}(x)<0, \forall t \in S ; f_{t}(x) \leq 0, \forall t \in W\right\}, F \neq \varnothing$ is characterized in the following cases:

- $S=\varnothing$ and $\left\{f_{t}, t \in W\right\} \subset \mathcal{C}$.

- $S \neq \varnothing$ and $\left\{f_{t}, t \in S \cup W\right\}$ is contained in either $\mathcal{A}$ or $\mathcal{S}$.

- $S \neq \varnothing,\left\{f_{t}, t \in W\right\} \subset \mathcal{C}$, and $\left\{f_{t}, t \in S\right\}$ is contained in either $\mathcal{S}$ or $\mathcal{M}$.

Concerning the containment of $F \neq \varnothing$ in the reverse-convex set $\mathbb{R}^{n} \backslash G_{j}$, with $G_{j}=\left\{x \in \mathbb{R}^{n} \mid h_{j}(x)<0\right\}$, it is characterized in the following cases:

- $\left\{f_{t}, t \in S \cup W ; h_{j}\right\}$ is contained in either $\mathcal{A}$ or $\mathcal{S}$.

- $\left\{f_{t}, t \in W\right\} \subset \mathcal{C}$ and $\left\{f_{t}, t \in S ; h_{j}\right\}$ is contained in either $\mathcal{S}$ or $\mathcal{M}$.

Obviously, the containment of $F \neq \varnothing$ in the reverse-convex set $\mathbb{R}^{n} \backslash \bigcup_{j \in J} G_{j}$, with $G_{j}=\left\{x \in \mathbb{R}^{n} \mid h_{j}(x)<0\right\}, j \in J$, is characterized if $F \subset \mathbb{R}^{n} \backslash G_{j}$ is characterized for all $j \in J$.

Finally, observe that the proofs of Propositions 6.1-6.3 are based upon the explicit construction of a linear representation of $F$, that is, $\eta$. From this representation it is possible to obtain the strict dual cone of $F \neq \varnothing$ just applying Corollaries 5.3 and 5.4 .

Example 6.1 Let $F=\left\{x \in \mathbb{R}^{n} \mid g_{t}(x)<b_{t}, \forall t \in S ; g_{t}(x) \leq b_{t}, \forall t \in W\right\}$ $\neq \varnothing$, with $g_{t}, t \in S \cup W$ as in Proposition 6.1. Then the strict dual cone of $F$ is $K^{<}=\operatorname{eco}\left(M^{<} \cup N^{<}\right)$, where

$$
M^{<}=\operatorname{eco}\left[\operatorname{coneco}\left\{\bigcup_{t \in S} \partial g_{t}\left(0_{n}\right) \times\left\{b_{t}\right\} ;\left(\begin{array}{c}
0_{n} \\
1
\end{array}\right)\right\} \backslash\left\{0_{n+1}\right\}\right]
$$

and

$$
N^{<}=\operatorname{eco}[\overbrace{\left(\begin{array}{l}
a \\
b
\end{array}\right) \in \bigcup_{t \in W} \partial g_{t}\left(0_{n}\right) \times\left\{b_{t}\right\}}^{\bigcup}\left\{\operatorname{coneco}\left\{\left(\begin{array}{l}
a \\
b
\end{array}\right),\left(\begin{array}{c}
0_{n} \\
1
\end{array}\right)\right\} \backslash \mathbb{R}_{+}\left\{\left(\begin{array}{l}
a \\
b
\end{array}\right)\right\}\right\}] .
$$

Similar expressions can be given for the strict dual cone of the solutions set of $\xi$, under the assumptions of Proposition 6.2 and 6.3, provided that $\xi$ is consistent. 


\section{References}

[1] W. Fenchel, A remark on convex sets and polarity, Communications du Séminaire Mathématique de l'Université de Lund, Supplement, (1952), 82-89.

[2] G. Fung, O.L. Mangasarian and J. Shavlik, Knowledge-based support vector machine classifiers, Neural Information Processing Systems 15, S. Becker, S. Thrun and K. Obermayer, editors, MIT Press, Cambridge, MA, 521-528.

[3] M.A. Goberna, V. Jornet and M.M.L. Rodriguez, On the characterization of some families of closed convex sets, Contributions to Algebra and Geometry, 43 (2002), 153-169.

[4] M.A. Goberna and M.M.L. Rodriguez, Linear systems containing strict inequalities via evenly convex hulls, European J. Operational Research, to appear.

[5] M.A. Goberna and Lopez, Linear Semi-infinite Optimization, Wiley Series in Mathematical Methods in Practice, John Wiley \& Sons, Chichester, 1998.

[6] M.A. Goberna, M. Larriqueta and V. Vera de Serio, On the stability of the boundary of the feasible set in linear optimization, Set-Valued Analysis, 11 (2003), 203-223.

[7] J. B. Hiriart-Urruty and C. Lemarechal, Convex Analysis and Minimization Algorithms, Volumes I and II, Springer-Verlag, Berlin- Heidelberg, 1993.

[8] V. Jeyakumar, Characterizing set containments involving infinite convex constraints and reverse-convex constraints, SIAM J. Optimization, 13 (2003) 947959 .

[9] V. Jeyakumar, Farkas' lemma: Generalizations, Encyclopedia of Optimization, Kluwer Academic Publishers, Dordrecht, The Netherlands, II (2001) 87- 91

[10] O.L. Mangasarian, Mathematical programming in data mining, Data Mining and Knowledge Discovery, 1 (1997), 183-201.

[11] O.L. Mangasarian, Set Containment characterization, J. of Global Optimization, 24 (2002), 473 - 480.

[12] O.L. Mangasarian, Nonlinear Programming, SIAM, Philadelphia, PA, 1994.

[13] Y.J. Zhu, Generalizations of some fundamental theorems on linear inequalities, Acta Math. Sinica, 16 (1966), 25-39. 